\title{
Primate-specific evolution of noncoding element insertion into PLA2G4C and human preterm birth
}

\author{
Jevon Plunkett ${ }^{1,2}$, Scott Doniger ${ }^{3}$, Thomas Morgan ${ }^{1,4}$, Ritva Haataja ${ }^{5}$, Mikko Hallman ${ }^{5}$, Hilkka Puttonen ${ }^{6}$, \\ Ramkumar Menon ${ }^{7,8}$, Edward Kuczynski ${ }^{9}$, Errol Norwitz ${ }^{9}$, Victoria Snegovskikh ${ }^{9}$, Aarno Palotie ${ }^{10,11,12,13}$, \\ Leena Peltonen ${ }^{10,11,12}$, Vineta Fellman ${ }^{14,15}$, Emily A DeFranco ${ }^{16}$, Bimal P Chaudhari ${ }^{17}$, John Oates ${ }^{18}$, \\ Olivier Boutaud ${ }^{18}$, Tracy L McGregor ${ }^{1}$, Jude J McElroy ${ }^{1}$, Kari Teramo ${ }^{6}$, Ingrid Borecki ${ }^{19}$, Justin C Fay ${ }^{20}$, \\ Louis J Muglia ${ }^{1,21^{*}}$
}

\begin{abstract}
Background: The onset of birth in humans, like other apes, differs from non-primate mammals in its endocrine physiology. We hypothesize that higher primate-specific gene evolution may lead to these differences and target genes involved in human preterm birth, an area of global health significance.

Methods: We performed a comparative genomics screen of highly conserved noncoding elements and identified PLA2G4C, a phospholipase A isoform involved in prostaglandin biosynthesis as human accelerated. To examine whether this gene demonstrating primate-specific evolution was associated with birth timing, we genotyped and analyzed 8 common single nucleotide polymorphisms (SNPs) in PLA2G4C in US Hispanic ( $\mathrm{n}=73$ preterm, 292 control), US White ( $n=147$ preterm, 157 control) and US Black ( $n=79$ preterm, 166 control) mothers.

Results: Detailed structural and phylogenic analysis of PLA2G4C suggested a short genomic element within the gene duplicated from a paralogous highly conserved element on chromosome 1 specifically in primates. SNPs rs8110925 and rs2307276 in US Hispanics and rs11564620 in US Whites were significant after correcting for multiple tests ( $p$ < 0.006). Additionally, rs11564620 (Thr360Pro) was associated with increased metabolite levels of the prostaglandin thromboxane in healthy individuals $(p=0.02)$, suggesting this variant may affect PLA2G4C activity.
\end{abstract}

Conclusions: Our findings suggest that variation in PLA2G4C may influence preterm birth risk by increasing levels of prostaglandins, which are known to regulate labor.

\section{Background}

A growing body of evidence supports genetic influences on preterm birth risk; however, few genes have been consistently associated with the disorder [1,2]. Investigators have typically focused on candidate genes selected based on predicted parturition physiology; however, this approach may be limited by the divergence in physiological mechanisms between humans and model organisms that have been typically studied. For example, while a rapid decline in progesterone plays a prominent role in initiating parturition in rodents and sheep, this

\footnotetext{
* Correspondence: louis.muglia@vanderbilt.edu

'Department of Pediatrics, Vanderbilt University School of Medicine Nashville, TN 37232, USA

Full list of author information is available at the end of the article
}

signal does not seem to precede human labor [3]. Other parturition-related traits, such as placental morphology and source of progesterone, also differ importantly in humans compared to model organisms typically studied and may limit what generalizations can be made [3].

Differences in parturition physiology between apes, including humans, and other mammals may have developed in response to uniquely human adaptations including relatively large human head size and narrow birth canal cross-sectional area [4]. Genes involved in parturition likely have evolved differentially along the human and/or higher primate phylogenetic lineages to decrease the length of gestation and alleviate the complications arising from such cephalopelvic constraints. As a result, the set of genes rapidly evolving on the human and/or 
higher primate lineage likely includes genes that play important roles in regulating parturition and potentially influence preterm birth risk. Consistent with our hypothesis, we identified $F S H R$ as having rapidly evolved by nucleotide substitution and as being associated with preterm birth risk across independent populations ([5] and (Plunkett J, Doniger S, Orabona G, Morgan T, Haataja R, Hallman $M$, Puttonen $H$, Menon $R$, Kuczynski E, Norwitz E et al: Evolutionary history of FSHR in human predicts role in birth time, submitted)).

In addition to nucleotide substitution, genomic rearrangements account for a substantial portion of genomic divergence among species. For example, Frazer et al. [6] and Wetterbom et al. [7] observed insertions and deletions frequently when comparing genome sequences among humans, chimpanzees and other primate species. Moreover, such rearrangements may account for a larger fraction of genomic divergence than nucleotide substitutions [7]. Rearrangements can lead to loss or acquisition of exons, splice sites and promoters, facilitating differences in expression patterns, such as those observed for transcript variants of CHRM3 and SFTPB with differing transposable element insertion events $[8,9]$. Hence, genomic rearrangement may contribute to rapid evolution along the human and/or higher primate lineages in response to unique physiological constraints.

We hypothesized that genes with genomic rearrangements departing from the ancestral state and occurring on the human and/or higher primate lineages may play important roles in birth timing and preterm delivery. Thus, we investigated association with preterm birth for common variants in a gene, $P L A 2 G 4 C$, which is expressed in the uterus [10] and involved in prostaglandin synthesis, suggesting a potential role in parturition, and in which we have identified a primate-specific insertion.

\section{Results}

\section{Evolutionary history of a primate-specific PLA2G4C noncoding element}

We identified genes showing evidence of rapid evolution along the human lineage, based on evidence from a comparative genomic screen of highly conserved noncoding elements as previously described [5]. Among the rapidly evolving genes emerging from our noncoding screen, PLA2G4C was identified as the most statistically significant human-lineage accelerated gene $(\mathrm{p}=2.2 \times$ $10^{-7}$, significant at $10 \%$ False Discovery Rate threshold) that was also included in a list of preterm birth candidate genes [11]. Because the reported deletion of PLA2G4C in cattle [12] contrasted with its presence in the 17-way MultiZ alignments [13] used to identify the gene as rapidly evolving (Figure $1 \mathrm{~A}$ ), we examined the history of this region in greater depth. We compared sequence surrounding the 130 base pair (bp) highly conserved noncoding element in intron 14 of PLA2G4C, located on chromosome 19q13.3, which strongly suggested the gene's designation as rapidly evolving along the human lineage, in comparison to other mammalian and primate genomes. From such comparisons, we determined that this $130 \mathrm{bp}$ element on human chromosome 19 was highly similar to a highly conserved noncoding element on human chromosome 1 (BLASTN 114/130 bp identical (87\%), BLAST Expect value (i.e. number of matches of this similarity likely to occur by chance alone) $=5 \times 10^{-38}$; Figure 1B). Subsequent analysis showed that the MultiZ alignments that we used in our comparative genomics screen had misaligned the human chromosome 19 element with sequences in other mammals which were orthologous to human chromosome 1 . When appropriate alignments were examined, we observed that the human chromosome 19 element was nearly identical in higher primate species (chimpanzee, gorilla, orangutan, macaque) examined, but absent in syntenic sequences in lower primates (lemur, bushbaby, tarsier) and other mammalian species. Chromosome 1 elements from higher primates are more similar to lower primates and other mammalian species than chromosome 19 elements (Figure 2). The chromosome 1 element occurs in the 5' untranslated region of RNF11, a gene involved in inflammatory signaling (UniProt KB) in mouse. Thus, a duplication of chromosome 1 noncoding element to chromosome 19 likely occurred before the last common ancestor between apes and macaque. A phylogenetic tree of coding sequences for PLA2G4C follows the expected mammalian phylogeny (Figure 3 ), suggesting that the duplication did not include coding sequences. Together these results suggest that neither element would qualify as rapidly evolving along the human lineage due to nucleotide substitution, but the chromosome 19 element may represent a primate-specific change meriting further study.

\section{Association with preterm birth}

Having identified PLA2G4C as a candidate gene for regulation of parturition timing, we tested variants in this gene for association with preterm birth in a case-control study involving diverse clinical populations. Because of recent data suggesting that heritability of preterm birth risk acts largely or exclusively through the maternal genome [14-16], we genotyped US Hispanic (73 preterm, 292 control), US Whites ( $\mathrm{n}=147$ preterm, 157 control) and US Black ( $\mathrm{n}=79$ preterm, 166 control) mothers for 14. SNPs in the PLA2G4C gene region (Additional file 1 Table S1). We were able to analyze 8 of these 14 SNPs that met our quality and frequency cut-off criteria (Additional file 1 Table S1). Power analysis for allelic association in each of these relatively limited populations modeling a relative risk of 2.0 for the high risk allele 
A

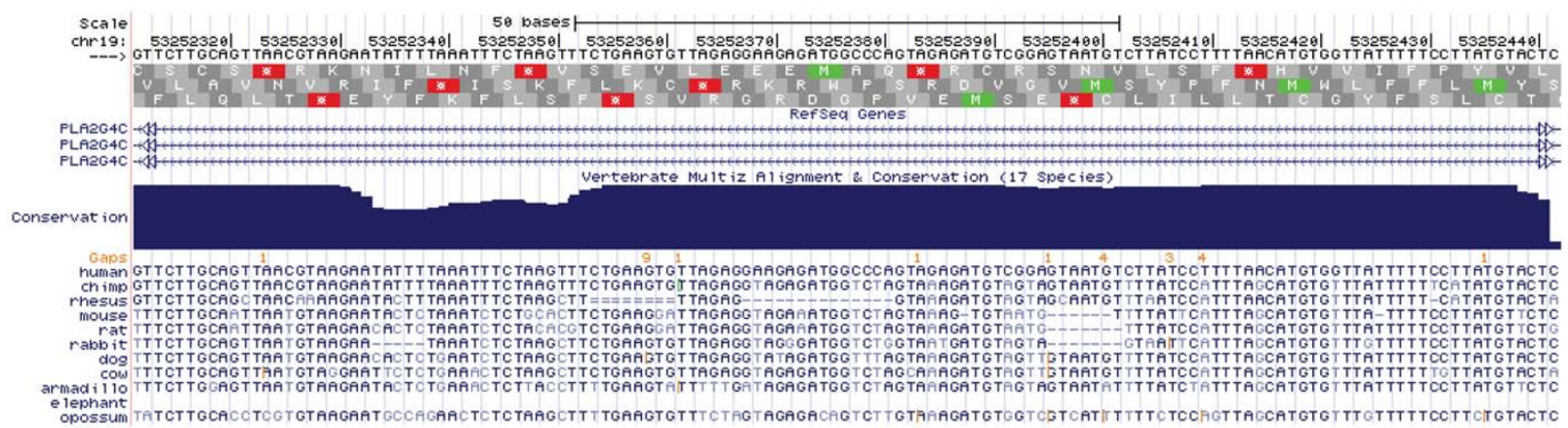

B

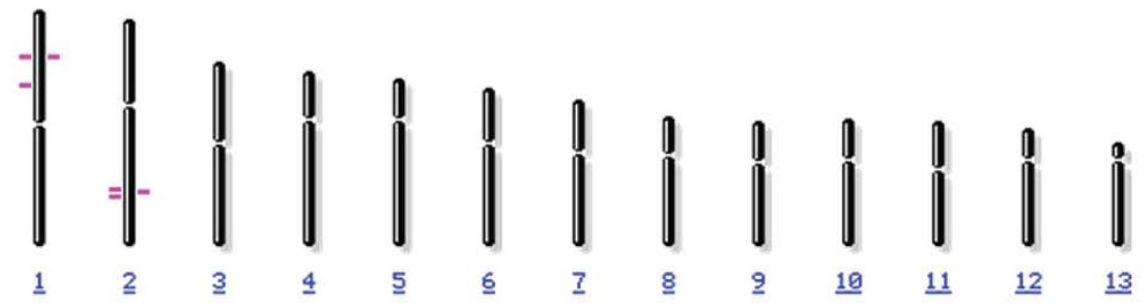

Figure 1 Genomic alignments suggest PLA2G4C noncoding element duplicated from another chromosome. MultiZ alignments used in the noncoding analysis from which we initially identified PLA2G4C as rapidly evolving include sequence for lower mammals, including cow, in which the gene is absent (Panel A). A BLASTN search of the element located in PLA2G4C intron 14 (red bar) (chromosome 19) that led to the gene's designation as rapidly evolving by nucleotide substitution revealed highly conserved noncoding elements (purple bars) on human chromosomes 1 and 2 (Panel B).

showed actual power for detection at $\mathrm{p}<0.05$ (not correcting for multiple tests) ranging from $51-86 \%$, and $\mathrm{p}<$ 0.006 (adjusting for 8 comparisons) ranging from 22 $62 \%$ depending upon high risk allele frequency (Additional file 2 Table S2). The results from these analyses include two SNPs, rs8110925 and rs2307276, in the US Hispanics and one in the US Whites, rs11564620, that were significant after correcting for 8 tests $(\mathrm{p}<0.006)$. Prior to constructing a meta-analysis, we performed a test for homogeneity across the three populations for each of these three SNPs. Two SNPs, rs8110925 and rs2307276, showed significant heterogeneity, implying population-specific effects. The heterogeneity p-value for rs11564620, in contrast, was not significant $(\mathrm{p}=0.21)$, so we performed a formal meta-analysis across populations. In this circumstance, the test for overall effect across populations resulted in $\mathrm{p}=0.02$ (Figure 4). Given the relatively limited number of studies and subjects, we went on to gain additional biological support by measuring prostaglandin levels (see below).

Additionally, 2, 3 and 4 SNP haplotypes containing SNPs rs8110925 and rs2307276 were significant in the US Hispanics after correcting for 18 haplotype comparisons ( $\mathrm{p}<$ 0.003), although not more significant than single SNP association findings (Additional file 3 Table S3, S4). 2 SNP haplotypes containing rs11564620 were moderately significant $(\mathrm{p}<0.05)$ in US Whites (Additional file 3 Table S3, Additional file 4 Table S4). Linkage disequilibrium (LD) among SNPs rs8110925, rs2307276, and rs11564620 was very low $\left(\mathrm{r}^{2}<0.1\right)$ in the three populations studied (Additional file 5 Figure S1), suggesting multiple independent associations were observed.

\section{Association with prostaglandin concentrations}

To test the potential functional effect of associated PLA2G4C variants on prostaglandin metabolism, we 


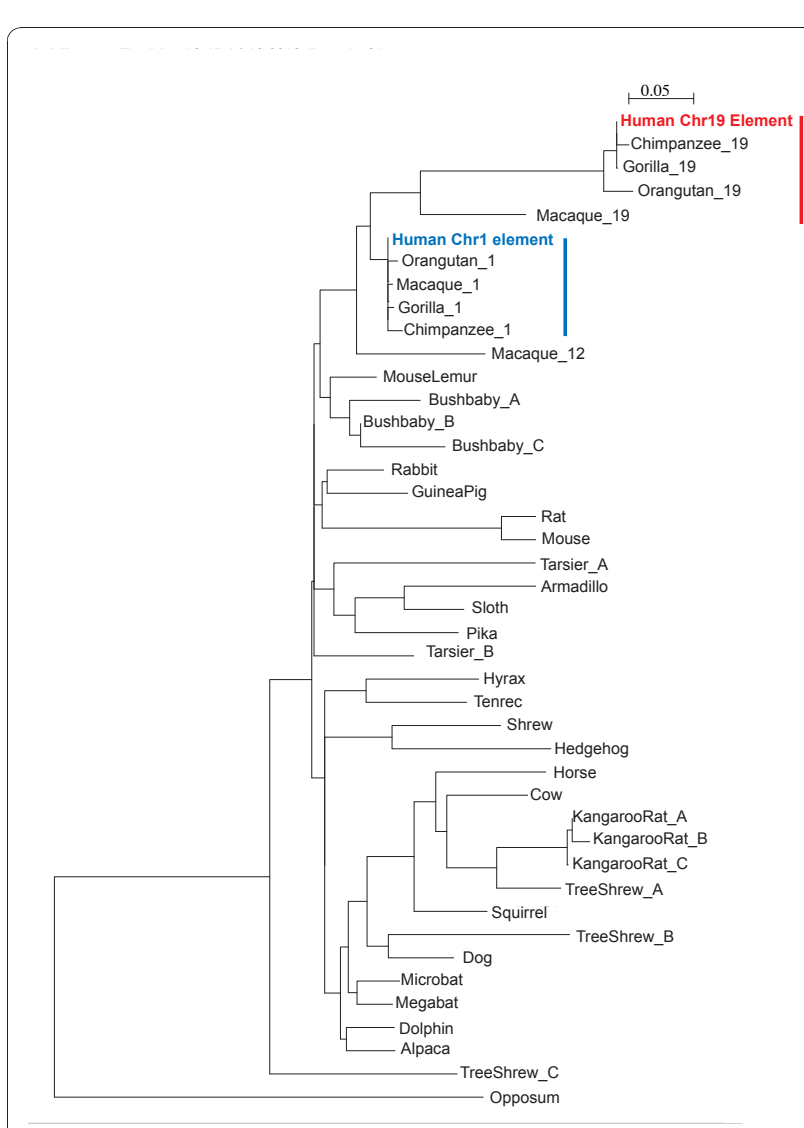

Figure 2 Phylogeny with sequences homologous to human chromosomes 19 noncoding element. Species name followed by a number indicates from which chromosome the sequence is derived or by a letter indicates that multiple copies homologous to the human chromosome 19 noncoding element were identified for that species. Sequences from lower primates and other mammalian species are more similar to higher primate sequences orthologous to human chromosome 1 noncoding element (indicated in blue) than sequences orthologous to human chromosome 19 noncoding element (indicated in red). A duplication of chromosome 1 noncoding element to chromosome 19 likely occurred before the last common ancestor between apes and macaque.

compared levels of metabolites of prostaglandin E2 (PGE), prostaglandin $\mathrm{I} 2$ (PGI) and thromboxane (11DTXB2) among genotype classes for associated SNPs rs8110925, rs2307276, and rs11564620 in healthy individuals using a two-sided Wald test (Additional file 6 Table S5). We hypothesized that these variants, particularly the coding region variant, would be associated with altered prostaglandin levels independent of pregnancy status. Of note, rs11564620, a nonsynonymous coding polymorphism, is associated with 11-DTXB2 levels $(\mathrm{p}=$ $0.04)$ despite the limited sample size available. The minor allele of rs11564620, present at approximately $10 \%$ frequency in US Whites, is associated with both risk for preterm birth and higher 11-DTXB2 levels (Wilcoxon one-sided $\mathrm{p}=0.02$; Figure 5).

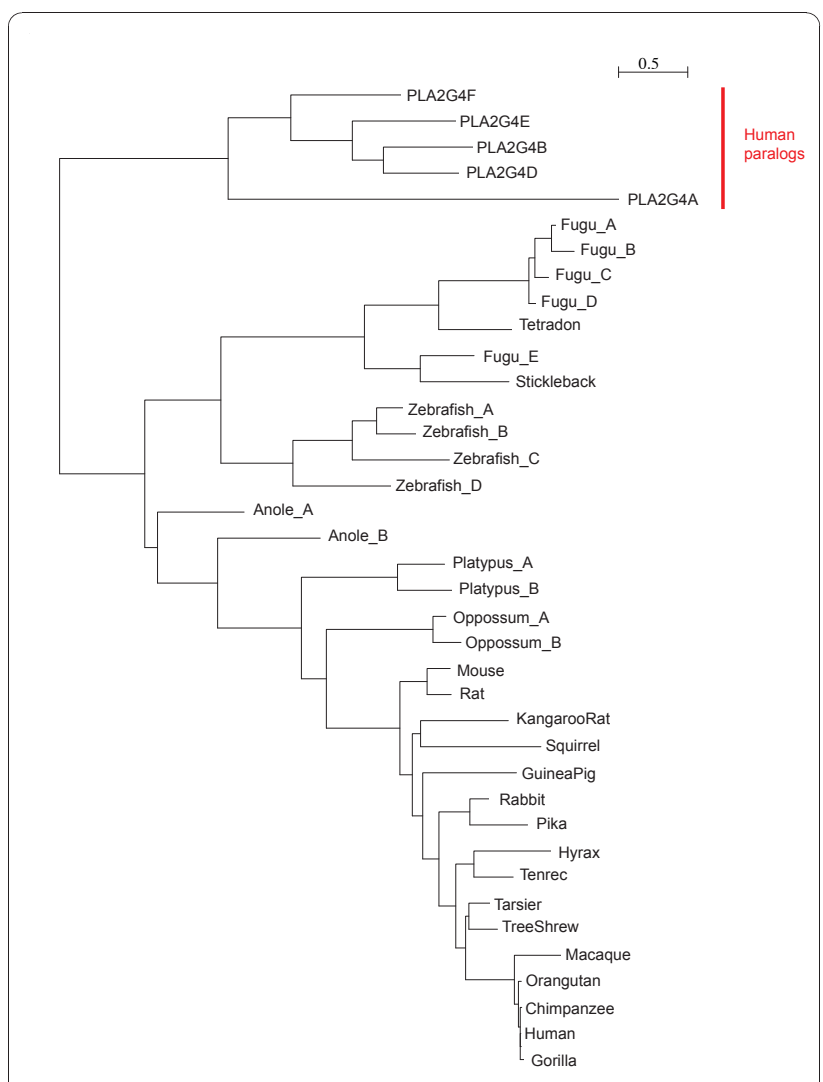

Figure 3 Phylogeny with coding sequences homologous to human PLA2G4C. Species name followed by a letter indicates that multiple copies homologous to human PLA2G4C were identified for that species. Phylogenetic tree of coding sequences follows expected relationships between species, suggesting that the duplication event of chromosome 1 sequence to chromosome 19 did not include coding sequence.

\section{Discussion}

Comparative genomic analysis is an attractive method for identifying genetic variation among species that may correlate with inter-specific phenotypic variation in fundamental processes such as parturition. Through such an analysis, we have identified a noncoding element in intron 14 of PLA2G4C on chromosome 19 representing a primate-specific change involving amplification and subsequent divergence but without increased nucleotide substitution. Having identified PLA2G4C as a candidate gene, we proposed that this duplicated element represents a primate-specific change with a potential regulatory role in human parturition.

We then tested our hypothesis by case-control association studies of preterm birth in several genetically diverse populations. Single SNP and haplotype association results implicated the role of SNPs rs8110925, rs2307276, and rs11564620 in preterm birth risk (Table 1 and Additional file 3 Table S3). The associated SNPs are located in an 8 kilobase $(\mathrm{kb})$ region of 


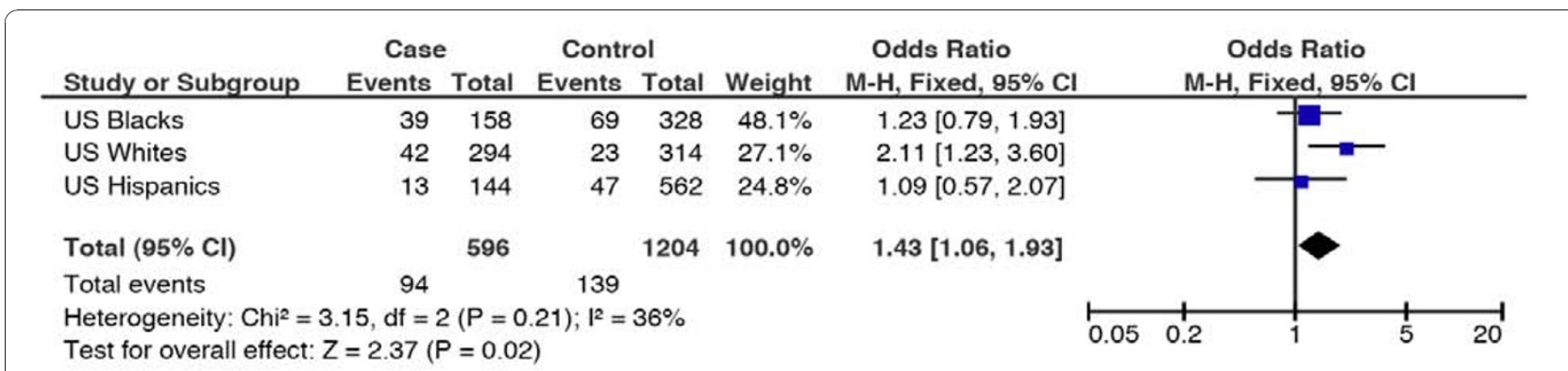

Note: Events refers to minor allele counts; patient sample size is half the total number of alleles.

Figure 4 Meta-analysis for US Hispanic, US White and US Black SNP association results for rs 11564620.

the 3' end of PLA2G4C, near the genomic element of interest (Figure 6), but show little LD with each other (Additional file 5 Figure S1) or other SNPs in PLA2G4C documented in the International HapMap Project database [17]. Of note, Polyphen [18] and SIFT [19] programs predict rs11564620, a nonsynonymous polymorphism in exon 13 resulting in a change in amino acid 360 from threonine to proline, to be possibly damaging to the protein structure (using protein sequence NP_003697 for PLA2G4C). This $8 \mathrm{~kb}$ region also includes coding sequence for aspartic acid 385 , one of the three amino acids that make up the putative active site of the enzyme [20], such that the proline substitution may alter the active site's physical conformation. Supporting the potential functional effect of

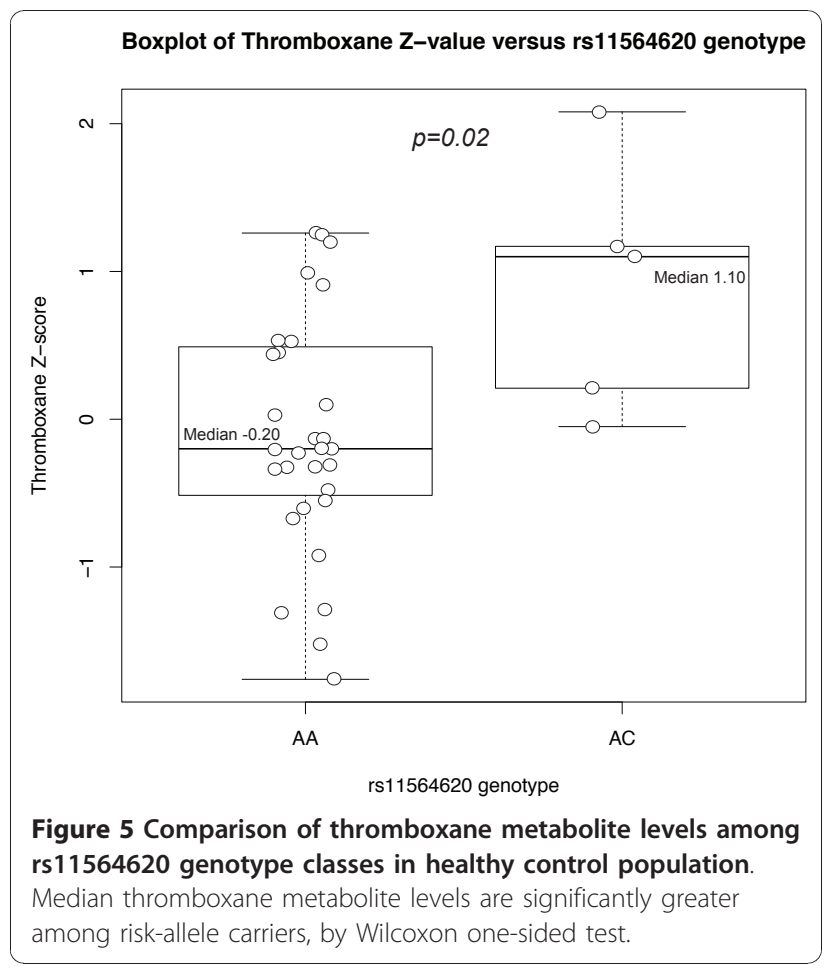

rs11564620, this polymorphism is associated with 11 -DTXB2 levels in healthy individuals $(\mathrm{p}=0.02$; Figure 5), with proline allele carriers having elevated thromboxane metabolite levels, compared to threonine homozygotes. Further suggesting functional importance of rs11564620, this SNP is also significantly associated ( $\mathrm{p}<0.0001$ ) with altered expression of NFATC2IP, a factor regulating cytokine expression in $\mathrm{T}$ cells located on chromosome 16 in quantitative trait databases for CEU populations http://scan.bsd.uchicago.edu/newinterface/about.html. Last, the evidence of modest deviation $(\mathrm{p}=0.04)$ from Hardy-Weinberg Equilibrium in the control US White population suggests that this variant may be under selection consistent with our hypothesis of selective pressure on genes involved in human parturition.

PLA2G4C encodes cytosolic phospholipase A2 gamma, which hydrolizes phospholipids from the cellular membrane to form free arachidonic acid, from which prostaglandins, including prostaglandins D, E, F, I2 (also known as prostacyclin), and thromboxane A2 are generated. Prostaglandins play an important role in parturition. Pharmacologically, prostaglandins are used to induce abortion, for cervical ripening, and labor induction and drugs inhibiting prostaglandin synthesis are successful in preventing preterm labor [21]. Levels of prostaglandins, including thromboxane $\mathrm{A} 2$, are elevated in pregnant compared to non-pregnant women, and in late (36 weeks) compared to early (20, 30 weeks) gestation [22], suggesting a link between prostaglandin abundance and parturition timing. Prostaglandins may facilitate labor by several mechanisms. These hormones are known uterotonic agents and also promote luteolysis and the onset of labor in species that exhibit progesterone withdrawal prior to birth [23]. Prostaglandins may also facilitate delivery by affecting placenta function, since thromboxane A2 induces platelet aggregation and acts as a vasoconstrictor [22]. Hence, higher prostaglandin levels than expected may initiate parturition prematurely and lead to preterm delivery. 
Table 1 Case-control association results for significant and suggestive SNPs in the PLA2G4C gene region tested across 3 independent populations.

\begin{tabular}{|c|c|c|c|c|c|c|c|}
\hline Population & SNP & Allele & Case Frequency & Control Frequency & Allele $p$-value & Genotype p-value & Allelic OR $(95 \% \mathrm{CI})$ \\
\hline \multirow{4}{*}{$\begin{array}{c}\text { US Hispanic } \\
\text { (73 cases, } 292 \text { controls) }\end{array}$} & rs8110925 & G & 0.18 & 0.085 & $7.92 \times 10^{-4} \mathrm{a}, \mathrm{b}$ & $5.66 \times 10^{-5} \mathrm{~b}$ & $2.4(1.4-4.1)$ \\
\hline & rs 2307276 & A & 0.11 & 0.036 & $5.45 \times 10^{-3} \mathrm{~b}$ & 0.01 & $3.2(1.6-6.5)$ \\
\hline & rs1366442 & A & 0.49 & 0.36 & 0.01 & 0.03 & $1.7(1.2-2.4)$ \\
\hline & rs11564620 & G & 0.09 & 0.08 & $0.55^{c}$ & $0.63^{c}$ & $1.1(0.6-2.1)$ \\
\hline \multirow{4}{*}{$\begin{array}{c}\text { US White } \\
\text { (147 cases, } 157 \text { controls) }\end{array}$} & rs8110925 & G & 0.058 & 0.057 & 0.92 & 0.59 & $1.0(0.5-2.0)$ \\
\hline & rs2307276 & A & 0.031 & 0.041 & 0.87 & 0.86 & $0.8(0.3-1.8)$ \\
\hline & rs1366442 & G & 0.4 & 0.42 & 0.5 & 0.02 & $0.9(0.6-1.2)$ \\
\hline & rs11564620 d & G & 0.14 & 0.07 & $6.98 \times 10^{-3}$ & $1.03 \times 10^{-3 \mathrm{~b}}$ & $2.1(1.2-3.6)$ \\
\hline \multirow{4}{*}{$\begin{array}{c}\text { US Black } \\
\text { (79 cases, } 166 \text { controls) }\end{array}$} & rs8110925 & G & 0.22 & 0.21 & $0.58^{e}$ & $0.83^{e}$ & $1.1(0.7-1.7)$ \\
\hline & rs2307276 & A & 0.093 & 0.14 & 0.22 & 0.49 & $0.6(0.3-1.2)$ \\
\hline & rs1366442 & G & 0.42 & 0.4 & 0.92 & 0.94 & $1.1(0.7-1.6)$ \\
\hline & rs11564620 & G & 0.25 & 0.21 & $0.47^{c}$ & $0.36^{c}$ & $1.2(0.8-1.9)$ \\
\hline
\end{tabular}

${ }^{a}$ Bolded numbers indicate $\mathrm{p}$-value $<0.05$.

${ }^{\mathrm{b}}$ Marker significant correcting for 8 tests $(p<0.006)$

'Same allele trends in same direction as US Whites.

${ }^{d} p=0.04$ for deviation from Hardy-Weinberg Equilibrium.

${ }^{\mathrm{e}} \mathrm{Same}$ allele trends in same direction as US Hispanics.

The PLA2G4C enzyme is the only cytosolic phospholipase A2 family member that is constitutively associated with the cellular membrane, the site of prostaglandin synthesis, rather than translocating to the membrane in response to calcium signaling [10]. Hence, dysregulation of PLA2G4C may alter prostaglandins levels independent of other parturition signals, such as oxytocin [24], that act via intracellular calcium signaling. For example, rs11564620 may contribute to a conformational change in the enzyme's active site, rendering it more active than

\section{fouthowhinit

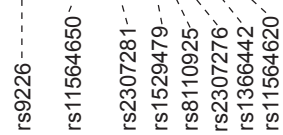

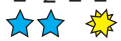

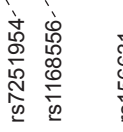

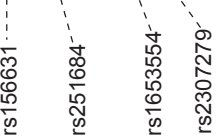

Figure 6 Overview of the SNPs tested in the PLA2G4C gene region. The gene structure for $P L A 2 G 4 C$ is represented by an arrow in which grey rectangles designate $3^{\prime}$ and $5^{\prime}$ untranslated regions and white rectangles designate coding exons. A red ellipse represents the primate-specific element of interest. Blue stars indicate rs2307276 and rs8110925, and yellow starburst indicates rs11564620, which is significant after multiple testing correction ( $p<$ 0.006) in US Hispanics and US Whites, respectively. usual and leading to increased synthesis of prostaglandins, as demonstrated by our observation of higher levels of thromboxane A2 in minor allele carriers for this polymorphism. Moreover, multiple splice isoforms of PLA2G4C exist, differing in transcript length, presence of certain exons and overlapping exons with different boundaries (AceView, NCBI, http://www.ncbi.nlm.nih.gov/IEB/ Research/Acembly/). As a result, variation in PLA2G4C may contribute to differences in tissue-specific expression or relative abundance of various PLA2G4C isoforms, potentially altering function. Further study of the region encompassing these SNPs, including the genomic element of interest, is needed to examine the mechanism by which variation in PLA2G4C influences birth timing.

Specialization within multi-gene families, like the large phospholipase A2 gene family, can create individualized functions among paralogous genes. For example, PLA2G4C has a continuous association with the cellular membrane, unlike other phospholipase A2 genes, potentially differentiating its role in prostaglandin synthesis from those of other family members. Genomic variation, such as the element insertion observed in PLA2G4C, may contribute to gene specialization, as demonstrated by divergence in PLA2G4C expression patterns in humans versus mice, who lack the element insertion and express PLA2G4C only in ovary and oocytes[25]. Specialized genes are potentially better therapeutic 
targets than gene products with multiple roles within cell, since pharmaceutically targeting such genes may lead to fewer side effects. As a result, PLA2G4C may be a useful target for designing novel therapies to prolong pregnancy and reduce the incidence of preterm birth.

\section{Conclusions}

A higher primate-specific noncoding element insertion into intron 14 of the phospholipase A2 gene PLA2G4C was identified, demonstrating the gene's rapid evolution along the higher primate lineage by genomic rearrangement. Results from our genetic analysis suggest common variation in PLA2G4C influences preterm birth risk. One of the variants associated with preterm birth is a nonsynonymous coding polymorphism, rs11564620, predicted to be potentially altering protein structure. This coding polymorphism is also associated with thromboxane levels, suggesting that genetic variation in PLA2G4C may increase risk for preterm birth by increasing levels of prostaglandins, which are known to regulate labor. By examining rapid evolution along human and higher primate lineages by genomic rearrangement, we have identified a novel gene associated with preterm birth. This approach can be readily applied to other traits differing among humans and/or higher primates and other species to aid in gene discovery.

\section{Methods}

\section{Genomic alignments to investigate evolutionary history of PLA2G4C}

Noting the deletion of PLA2G4C reported in the Taurine Cattle genome [12] contrasted with its presence in the 17-way MultiZ alignments [13] we used to identify the gene as rapidly evolving (analysis conducted Spring 2007 and presented in detail in (Plunkett J, Doniger S, Orabona G, Morgan T, Haataja R, Hallman M, Puttonen H, Menon R, Kuczynski E, Norwitz E et al: Evolutionary history of FSHR in human predicts role in birth time, submitted)), we examined the history of this region in greater depth. We extracted sequence surrounding the $130 \mathrm{bp}$ highly conserved noncoding element (human chromosome 19: 48,560,500 -48,560,630; hg19 genome build) which largely contributed to our designation of PLA2G4C as rapid evolving along the human lineage. A BLASTN search of the element revealed highly identical conserved noncoding elements on human chromosomes 1 (87\% identity) and 2 (85\% identity) (Figure 1B). We compared the human chromosome 19 and chromosome 1 sequences to 31 eutherian mammalian genomes using Ensembl Genomic alignments (accessed September 2009), and ClustalW alignment, and to specific primate genomes using BLASTN searches of human, chimpanzee, gorilla, orangutan, macaque, and bushbaby genomes (accessed September
2009). We then reconstructed history of the element by creating phylogenies using maximum likelihood with sequences homologous to the human chromosome 19 element (Figure 2) and coding sequences homologous to human PLA2G4C (Figure 3).

\section{Human subjects}

Study subjects were enrolled for genetic analysis by methods approved by Institutional Review Boards/Ethics Committees at each participating institution. Informed consent was obtained for all participants. Mothers with preterm birth were included if the birth was spontaneous (non-iatrogenic), singleton, had no obvious precipitating stimulus (trauma, infection, drug use), and was less than 37 weeks (Yale University; New York University) or 36 weeks (Centennial Hospital, Nashville, TN) of completed gestation. Control mothers were included if they had delivered two or more children at 37 weeks or later spontaneously. Healthy volunteers were recruited at Vanderbilt University for studies of prostaglandin metabolism. DNA from blood or saliva was prepared by standard methods. Race/ethnicity was assigned by selfreport. All specimens were linked with demographic and medical data abstracted from maternal/neonatal records. DNA from blood or saliva was prepared by standard methods. Maternal age did not differ between cases and controls in the different populations $(27.3 \mathrm{y}$ vs. $28.4 \mathrm{y}$, $\mathrm{p}=0.10$ US White; 25.3 y vs. $25.2 \mathrm{y}, \mathrm{p}=0.88$ US Black; 26.0 y vs. 25.0 y, p $=0.20$ US Hispanic).

\section{Prostaglandin metabolite levels}

For individuals enrolled in the prostaglandin study, urine was collected by standard methods. Levels of the urinary metabolites of prostaglandin E (PGE), prostaglandin I (PGI) and thromboxane (11-DTXB2) were quantified by mass spectrometry and normalized to creatinine levels, an indicator of renal function, in 44 healthy control individuals of Black, Hispanic or White race (median age $29,60 \%$ male, $77 \%$ White).

\section{Genotyping}

We genotyped 14 SNPs spanning the PLA2G4C gene region (Additional file 1 Table $\mathrm{S} 1$ ) on human chromosome 19 in cohorts of US Hispanics ( $\mathrm{n}=73$ preterm, 292 control mothers), US Whites $(\mathrm{n}=147$ preterm, 157 control mothers) and US Blacks ( $\mathrm{n}=79$ preterm, 166 control mothers). For SNP selection, data from the HapMap Release 27 CEU population was examined in the Haploview program [26], using tagger and haplotype block functions, to identify regions of high LD. We selected 1 SNP per haplotype block, defined using the D' confidence interval method [27], having the highest minor allele frequency (MAF) in the CEU population for genotyping. We also included coding 
SNPs and other noncoding SNPs to improve coverage of conserved elements contributing to the gene's designation as "rapidly evolving." This selection scheme resulted in approximately 35\% coverage of the gene region at $r^{2} \geq 0.8$. SNPs showing evidence of association in one or more cohort $(\mathrm{p}<0.01 ; \mathrm{n}=4)$ were then genotyped in healthy individuals on whom data on their concentrations of several prostaglandin metabolites was available to examine potential functional effects of the variants. All SNPs were genotyped using the Sequenom iPLEX massARRAY technology (Sequenom, San Diego, CA).

\section{Data Analysis}

Data cleaning and analysis was performed with Wholegenome Association Study Pipeline (WASP) [28] and PLINK [29]. We excluded individuals based on genotyping quality ( $<90 \%$ call rate) and SNPs based on the following criteria: not in Hardy-Weinberg Equilibrium in controls $\left(\mathrm{p}<0.001 \chi^{2}\right.$ test), $<90 \%$ genotype call rate, MAF < 0.01). Linkage disequilibrium among SNPs tested was determined using the Haploview program [26]. We chose this Hardy-Weinberg threshold for two reasons. First, we hypothesize this locus is under selective pressure which could result in some deviation from HWE in the control population. Second, samples with $\mathrm{p}<0.001$ also had low genotype call rates, suggesting genotyping error, while those with $\mathrm{p}>0.001$ had high call rates. For significant SNPs, the Hardy-Weinberg deviation was greater than 0.05 unless otherwise indicated. We corrected for multiple testing using the simpleM method [30], which estimates the number of independent tests, given the LD relationships among SNPs, used to obtain a Bonferroni-corrected critical value.

Our analysis considered preterm birth affection status (i.e. delivery $<37$ weeks) as a binary trait, comparing frequencies between case and control groups of alleles and genotypes by $\chi^{2}$ test. Sliding windows of 2,3 and 4. SNP haplotypes also were compared between cases and controls [29]. Meta-analysis of data for significant SNPs was done using the Mantel-Haenszel method, after successfully passing the test of homogeneity.

To test the potential functional effect of associated PLA2G4C variants on prostaglandin metabolism, we examined the levels of PGE, PGI, and 11-DTXB2, standardized to normal distributions $(\mu=0, \sigma=1)$, as quantitative traits. A Wald test was performed to compare the mean phenotype between different allele or genotype classes for associated SNPs. We also tested whether rs11564620 risk-allele carriers had higher prostaglandin levels than noncarriers, by comparing the 11-DTXB2 value distribution among genotype classes with box plots and one-sided Wilcoxon nonparametric test performed in R [31].

\section{Additional material}

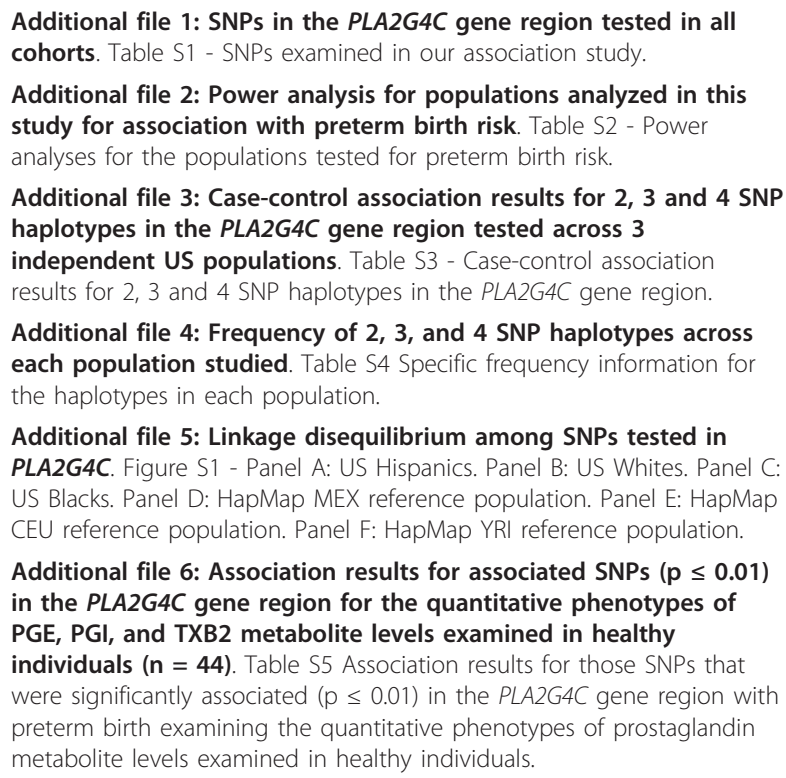

Additional file 4: Frequency of 2, 3, and 4 SNP haplotypes across each population studied. Table 54 Specific frequency information for the haplotypes in each population.

Additional file 5: Linkage disequilibrium among SNPs tested in PLA2G4C. Figure S1 - Panel A: US Hispanics. Panel B: US Whites. Panel C: US Blacks. Panel D: HapMap MEX reference population. Panel E: HapMap CEU reference population. Panel F: HapMap YRI reference population.

Additional file 6: Association results for associated SNPs ( $p \leq 0.01$ ) in the PLA2G4C gene region for the quantitative phenotypes of PGE, PGI, and TXB2 metabolite levels examined in healthy individuals $(\mathbf{n}=\mathbf{4 4})$. Table S5 Association results for those SNPS that were significantly associated $(p \leq 0.01$ ) in the PLA2G4C gene region with preterm birth examining the quantitative phenotypes of prostaglandin metabolite levels examined in healthy individuals.

\section{Acknowledgements}

We thank Cara Sutcliffe and Rachel Wiseman in the DNA Resources Core at Vanderbilt University Medical Center for their assistance with genotyping. This work was supported by grants from the Children's Discovery Institute at Washington University School of Medicine and St. Louis Children's Hospital awarded to JF and LJM and from the March of Dimes awarded to LJM and EN. This research was also supported by T32 GM081739 from the National Institute of General Medical Science and the Mr. and Mrs. Spencer T. Olin Fellowship for Women in Graduate Study at Washington University in St. Louis awarded to JP, a grant from the Sigrid Juselius Foundation awarded to $\mathrm{MH}$, a grant from the Signe and Ane Gyllenberg foundation to VF and grants from the Academy of Finland to $\mathrm{RH}$ and $\mathrm{MH}$. TM was supported by a Turner-Hazinski grant award from Vanderbilt University.

\section{Author details}

${ }^{1}$ Department of Pediatrics, Vanderbilt University School of Medicine, Nashville, TN 37232, USA. ${ }^{2}$ Human and Statistic Genetics Program, Washington University School of Medicine, St. Louis, MO 63110, USA. ${ }^{3}$ Computational Biology Program, Washington University School of Medicine, St. Louis, MO 63108, USA. ${ }^{4}$ Center for Human Genetics Research, Vanderbilt University School of Medicine, Nashville, TN 37232, USA. ${ }^{5}$ Institute of Clinical Medicine, Department of Pediatrics, University of Oulu, Oulu 90014, Finland. ${ }^{6}$ Departments of Obstetrics and Gynecology, University Central Hospital, Helsinki 00290, Finland. ${ }^{7}$ The Perinatal Research Center, Nashville, TN 37203. ${ }^{8}$ Department of Epidemiology, Rollins School of Public Health, Emory University, Atlanta, GA, 30322, USA. 'DDepartment of Obstetrics, Gynecology, and Reproductive Sciences, Yale University School of Medicine, New Haven, CT 06520, USA. ${ }^{10}$ Biomedicum Helsinki Research Program in Molecular Medicine, University of Helsinki, Helsinki 00290, Finland. ${ }^{11}$ The Finnish Genome Center, University of Helsinki, Helsinki 00290, Finland. ${ }^{12}$ The Broad Institute of MIT and Harvard, Cambridge, Massachusetts 02142, USA.

${ }^{13}$ Wellcome Trust Sanger Institute, Cambridge CB10 1SA, UK. ${ }^{14}$ Department of Pediatrics, Lund University, Lund 22185, Sweden. ${ }^{15}$ Department of Pediatrics, University of Helsinki, Helsinki 00290, Finland. ${ }^{16}$ Department of Obstetrics and Gynecology, University of Cincinnati College of Medicine, Cincinnati, $\mathrm{OH}$ 45267, USA. ${ }^{17}$ Department of Pediatrics, Washington 
University School of Medicine, St. Louis, MO 63110, USA. ${ }^{18}$ Division of Clinical Pharmacology, Vanderbilt University School of Medicine, Vanderbilt University, Nashville, TN 37232, USA. ${ }^{19}$ Division of Statistical Genomics, Washington University School of Medicine, St. Louis, MO 63108, USA. ${ }^{20}$ Department of Genetics and Center for Genome Sciences, Washington University School of Medicine, St. Louis, MO 63108, USA. ${ }^{21}$ Department of Molecular Physiology and Biophysics, Vanderbilt University School of Medicine, and Vanderbilt Kennedy Center for Human Development, Vanderbilt University, Nashville, TN 37232, USA.

\section{Authors' contributions}

$J P, S D, T M, J O, O B$, JF and LM designed research; RH, MH, HP, RM, EK, EN, VS, $A P, L P, V F, E D, B C, J O, O B, K T$ and $L J M$ contributed new reagents/analytic tools; JP, SD, GO performed research; JP, SD, JJM, TLM, TM, OB, JF and LJM analyzed data; and JP, MH, EO, JO, KT, IB, JF, TM, and LJM wrote the paper. All authors read and approved the final manuscript.

Received: 22 July 2010 Accepted: 24 December 2010 Published: 24 December 2010

\section{References}

1. Chaudhari BP, Plunkett J, Ratajczak CK, Shen TT, DeFranco EA, Muglia LJ: The genetics of birth timing: insights into a fundamental component of human development. Clin Genet 2008, 74(6):493-501.

2. Plunkett J, Muglia L: Genetic contributions to preterm birth: Implications from epidemiological and genetic association studies. Ann Med 2008, 40(3):167-179.

3. Mitchell BF, Taggart MJ: Are animal models relevant to key aspects of human parturition? Am J Physiol Regul Integr Comp Physiol 2009, 297(3) R525-545.

4. Rosenberg K, Trevathan W: Birth, obstetrics and human evolution. BJOG 2002, 109(11):1199-1206.

5. Fay JC, Wyckoff GJ, Wu Cl: Positive and negative selection on the human genome. Genetics 2001, 158(3):1227-1234.

6. Frazer KA, Chen X, Hinds DA, Pant PV, Patil N, Cox DR: Genomic DNA insertions and deletions occur frequently between humans and nonhuman primates. Genome Res 2003, 13(3):341-346.

7. Wetterbom A, Sevov M, Cavelier L, Bergstrom TF: Comparative genomic analysis of human and chimpanzee indicates a key role for indels in primate evolution. J Mol Evol 2006, 63(5):682-690.

8. Huh JW, Kim YH, Lee SR, Kim H, Kim DS, Kim HS, Kang HS, Chang KT: Gain of new exons and promoters by lineage-specific transposable elementsintegration and conservation event on CHRM3 gene. Mol Cells 2009, 28(2):111-117.

9. Lee JR, Huh JW, Kim DS, Ha HS, Ahn K, Kim YJ, Chang KT, Kim HS: Lineage specific evolutionary events on SFTPB gene: Alu recombinationmediated deletion (ARMD), exonization, and alternative splicing events. Gene 2009, 435(1-2):29-35.

10. Tithof PK, Roberts MP, Guan W, Elgayyar M, Godkin JD: Distinct phospholipase A2 enzymes regulate prostaglandin E2 and F2alpha production by bovine endometrial epithelial cells. Reprod Biol Endocrinol 2007, 5:16.

11. Committee on Understanding Premature Birth and Assuring Healthy Outcomes: Preterm Birth: Causes, Consequences, and Prevention. Washington, D.C.: The National Academies Press; 2006.

12. Elsik CG, Tellam RL, Worley KC, Gibbs RA, Muzny DM, Weinstock GM, Adelson DL, Eichler EE, Elnitski L, Guigo R, et al: The genome sequence of taurine cattle: a window to ruminant biology and evolution. Science 2009, 324(5926):522-528.

13. Blanchette M, Kent WJ, Riemer C, Elnitski L, Smit AF, Roskin KM, Baertsch R, Rosenbloom K, Clawson H, Green ED, et al: Aligning multiple genomic sequences with the threaded blockset aligner. Genome Res 2004, 14(4):708-715.

14. Kistka ZA, DeFranco EA, Ligthart L, Willemsen G, Plunkett J, Muglia $\sqcup$, Boomsma Dl: Heritability of parturition timing: an extended twin design analysis. Am J Obstet Gynecol 2008, 199(1), 43 e41 45.

15. Plunkett J, Feitosa MF, Trusgnich M, Wangler MF, Palomar L, Kistka ZA, DeFranco EA, Shen TT, Stormo AE, Puttonen $H$, et al: Mother's genome or maternally-inherited genes acting in the fetus influence gestational age in familial preterm birth. Hum Hered 2009, 68(3):209-219.
16. Wilcox AJ, Skaerven R, Lie RT: Familial patterns of preterm delivery: maternal and fetal contributions. Am J Epidemiol 2008, 167(4):474-479.

17. The International HapMap Consortium: A second generation human haplotype map of over 3.1 million SNPs. Nature 2007, 449(7164):851-861

18. Ramensky V, Bork P, Sunyaev S: Human non-synonymous SNPs: server and survey. Nucleic Acids Research 2002, 30(17):3894-3900.

19. $\mathrm{Ng}$ PC, Henikoff S: SIFT: predicting amino acid changes that affect protein function. Nucleic Acids Research 2003, 31(13):3812-3814.

20. Underwood KW, Song C, Kriz RW, Chang XJ, Knopf JL, Lin LL: A novel calcium-independent phospholipase A2, CPLA2-gamma, that is prenylated and contains homology to CPLA2. J Biol Chem 1998, 273(34):21926-21932.

21. Khan AH, Carson RJ, Nelson SM: Prostaglandins in labor-a translational approach. Front Biosci 2008, 13:5794-5809.

22. Hayashi M, Inoue T, Hoshimoto K, Hirabayashi $H$, Negishi $H$, Ohkura T: The levels of five markers of hemostasis and endothelial status at different stages of normotensive pregnancy. Acta Obstet Gynecol Scand 2002, 81(3):208-213.

23. Gross G, Imamura T, Vogt SK, Wozniak DF, Nelson DM, Sadovsky Y, Muglia LJ: Inhibition of cyclooxygenase-2 prevents inflammationmediated preterm labor in the mouse. Am J Physiol Regul Integr Comp Physiol 2000, 278(6):R1415-1423.

24. Burns PD, Mendes JO Jr, Yemm RS, Clay CM, Nelson SE, Hayes SH, Silvia WJ: Cellular mechanisms by which oxytocin mediates ovine endometrial prostaglandin F2alpha synthesis: role of $\mathrm{G}$ (i) proteins and mitogenactivated protein kinases. Biol Reprod 2001, 65(4):1150-1155.

25. Vitale A, Perlin J, Leonelli L, Herr J, Wright P, Digilio L, Coonrod S: Mouse CPLA2[gamma], a novel oocyte and early embryo-abundant phospholipase A2 gamma-like protein, is targeted to the nuclear envelope during germinal vesicle breakdown. Developmental Biology 2005, 282(2):374-384.

26. Barrett JC, Fry B, Maller J, Daly MJ: Haploview: analysis and visualization of LD and haplotype maps. Bioinformatics 2005, 21(2):263-265.

27. Gabriel SB, Schaffner SF, Nguyen H, Moore JM, Roy J, Blumenstiel B, Higgins J, DeFelice M, Lochner A, Faggart M, et al: The structure of haplotype blocks in the human genome. Science 2002, 296(5576):2225-2229.

28. Hafler DA, Compston A, Sawcer S, Lander ES, Daly MJ, De Jager PL, de Bakker PI, Gabriel SB, Mirel DB, Ivinson AJ, et al: Risk alleles for multiple sclerosis identified by a genomewide study. N Engl I Med 2007, 357(9):851-862.

29. Purcell S, Neale B, Todd-Brown K, Thomas L, Ferreira MA, Bender D, Maller J, Sklar P, de Bakker Pl, Daly MJ, et al: PLINK: a tool set for whole-genome association and population-based linkage analyses. Am J Hum Genet 2007, 81(3):559-575.

30. Gao X, Becker LC, Becker DM, Starmer JD, Province MA: Avoiding the high Bonferroni penalty in genome-wide association studies. Genet Epidemiol 2009, 34(1):100-105.

31. R Development Core Team: R: A language and environment for statistical computing. R Foundation for Statistical Computing; 2009.

\section{Pre-publication history}

The pre-publication history for this paper can be accessed here: http://www.biomedcentral.com/1755-8794/3/62/prepub

doi:10.1186/1755-8794-3-62

Cite this article as: Plunkett et al:: Primate-specific evolution of noncoding element insertion into PLA2G4C and human preterm birth. BMC Medical Genomics 2010 3:62. 